\title{
Factor structure and normative data of the Sinhalese version of self reported Strength and Difficulties Questionnaire (SDQ) for adolescents
}

\author{
S Perera, E Thalagala, S H Chandrarathna, T C Agampodi, D B Nugegoda, S B Agampodi \\ (Index words: strength and difficulties questionnaire, Sinhalese version, adolescents, factor structure, norma- \\ tive data)
}

\begin{abstract}
Objectives To describe the normative data, factor structure and the internal consistency of the Sinhalese, self reported version of SDQ for adolescents.

Methods The present study was carried out in eight provinces in Sri Lanka. A sample of school going adolescents aged 12-16 years were selected from Sinhalese medium schools using a multi stage cluster sampling technique with probability proportionate to size. The component structure of the SDQ was examined using principal component analysis. Normative banding and the cut off values were determined, based on the distribution of raw data in this non-clinical sample.

Results The study sample consisted of 535 (45.3\%) boys and 645 (54.5\%) girls. Mean total difficulty score for girls $(10.66 \pm 5.440)$ was significantly $(p=0.014)$ higher than that for boys $(9.93 \pm 4.671)$. Mean scores for emotional (2.97 \pm 2.009$)$, conduct $(2.11 \pm 1.755)$ and peer $(2.24 \pm 1.760)$ subscales were also significantly higher among girls than that of boys $(2.7 \pm 1.915,1.78 \pm 1.406$ and $2.04 \pm 1.383$ respectively). In the factor analysis, only factor that was loaded with all five items in the original subscale was "emotional" and the loading values were more than .445 for all five items. None of the other extracted factors contained more than 3 items from one subscale. Internal consistency (Cronbach's alpha) for total difficulties was satisfactory in the total sample as well as in boys and girls separately (>.71). However, in all five subscales, Chrobach's alpha was less than six, showing a low homogeneity.
\end{abstract}

Conclusions Factor structure and internal consistency of the current Sinhalese version of the self reported SDQ is not satisfactory. Revalidation of this version of SDQ is recommended to capture the intended objectives of the SDQ.

Ceylon Medical Journal 2013; 58: 66-71

\section{Introduction}

Strength and Difficulties Questionnaire (SDQ) is one of the most widely used instruments for screening behavioural problems in children. In 1997, Robert Goodman presented this short assessment instrument to the scientific community [1]. The SDQ is a brief behavioural screening questionnaire that provides a balanced coverage of children's and young people's behaviour, emotions, and relationships with good discriminate and predictive validity. The SDQ has parent reported, teacher reported and self reported versions for several age categories. This instrument is used by psychologists, community mental health services, school health services and epidemiologist throughout the world and has been translated to many languages. The SDQ website contains 75 versions of this instrument [2]. The original English version as well as translated versions of the questionnaire has been shown as a valid and reliable tool for screening behavioural problems in children. It is recommended to study the normative values and psychometric properties of different versions of the SDQ to decide cut off values for different populations. In almost all translated versions of SDQ, normative values and psychometric properties have been studied and published. The cut off values for normal, borderline and abnormal bands of mental health problems are shown to vary according to the normative data of the given translated version.

The Sinhalese version of self reported SDQ for adolescents aged 11-17 years in Sri Lanka has been translated and validated [3,4]. This Sinhalese version of SDQ has been used in studies in Sri Lanka too $[5,6]$. Previous authors suggested that the original cut off values proposed by Goodman should be used with the Sinhalese version $[3,4]$. However, these cut off values were not based on Sri Lankan normative data. Observations of the results of the national sample and few other studies carried out in Sri Lanka raised the question whether the cut off values are valid for the Sinhalese version. This question was difficult to answer based on the data published in the validation paper, because the normative data for the

Department of Community Medicine, Faculty of Medicine and Allied Sciences, University of Rajarata, Sri Lanka.

Correspondence: SBA, e-mail: <sunethagampodi@yahoo.com>. Received 27 April 2012 and revised version accepted 12 March 2013. Competing interests: none declared. 
Sinhalese population was not reported. Further, factor structure and the internal consistency of the Sinhalese version of the SDQ have not been published, although it has been reported that in a sample drawn from Colombo, these parameters were satisfactory [3]. The purpose of the present paper is to present the psychometric characteristics and the normative data for the Sinhalese version of the self reported version of the SDQ based on a nationally representative sample of schooling adolescents.

\section{Methods}

Sri Lanka has a 5 million adolescent population [7]. Of these, at least $86 \%$ complete their post primary education. Primary enrolment in education is $100 \%$ and 97\% complete primary education [8]. This study was based on the assumption that the schooling adolescents are a representative sample of adolescents. Aim of this study was to estimate normative data and assess the psychometric properties of the SDQ.

Adolescent school children in Sinhala medium schools from eight provinces were included as the study population. Northern province was excluded from the sampling frame due to security situations prevailing at the time of study. We also assumed that the effect will be minimal because there were only 16 Sinhala medium schools in the Northern province compared to 6779 in other provinces [9].

Multi stage cluster sampling procedure was used to select the study participants. A classroom with an average of 30 students was selected as the cluster. It was estimated that a minimum of 42 clusters $(n=1260)$ were required to estimate normative data in this study population, with a .05 precision and 95\% confidence limits. For content analysis of the SDQ the proposed sample size was 300 participants. In the first stage of sampling, districts to obtain clusters were selected using the probability proportionate to size sampling technique. In each selected district, schools with students in grade 8, 9 and 10 were selected and listed for the next stage of sampling. From these lists, schools were selected using probability proportionate to size sampling. All principals of selected schools were contacted to obtain permission to carry out this study. From each school, clusters from eligible class rooms were selected using convenient sampling to minimise the disturbances to classroom activities. Approval to carry out this study was obtained from the Department of Education and of the selected schools.

Three trained, MBBS qualified investigators (first three authors of this study) participated in the data collection, where they explained the procedure to participants in the classrooms. The self reported Sinhalese version of SDQ for adolescent 11-17 years old, available in www.sdqinfo.com was used in the study. Even though the expected cluster size was 30, the whole classroom participated in the study by filling the questionnaire, to minimise ethical problems. Since the objective was to analyse the Sinhalese version of the questionnaire, all participants, whose mother tongue was not Sinhalese were removed from the analysis. In small schools with less than 20 children in a classroom, two classes were combined to form a single cluster.

Data handling and statistical analysis was carried out using SPSS software version 17. Normative data for the total sample was calculated according to the instructions given in SDQ website. Mean and standard deviations of total score and five subscales were calculated separately. These descriptive data was further analysed according to age and sex. Internal consistency of the instrument was analysed using Chronbach's alpha coefficient for the total score and for subscales. The component structure of the SDQ was examined using principal component analysis (PCA), with all 25 items of SDQ in the model. In the first stage, optimal number of components were selected using eigenvalue of more than one. In the second step we forcefully restricted the number of factors extracted in the model to five, to be compatible with five subscales in the original questionnaire. To maximise the loading of factors, rotation was allowed up to 25 times. Based on the hypothesis that underlying factors (subscales) are independent, varimax rotation was selected. Absolute coefficient values below 0.3 in the model were suppressed for better interpretation of factors extracted and variables loaded for each factor.

\section{Results}

A total of 1346 adolescents participated in the study. We excluded 166 (12.3\%) questionnaires due to missing data in one or several items in the SDQ. The study sample consisted of 535 (45.3\%) boys and 645 (54.5\%) girls. Composition of the age categories was; $12-15$ years 841 (72.8\%) and $15-16$ years 313 (27.2\%).

Mean and standard deviations for total scores and the gender is showen in Table 1. Mean total difficulty score for girls (10.66 \pm 5.440$)$ was significantly $(p=.014)$ higher than that for boys $(9.93 \pm 4.671)$. Mean scores for emotional (2.97 \pm 2.009$)$, conduct (2.11 \pm 1.755$)$ and peer (2.24 \pm 1.760$)$ subscales were also significantly higher among girls than that of boys $(2.7 \pm 1.915,1.78 \pm 1.406$ and $2.04 \pm 1.383$ respectively).

There was a main effect of age on hyperactivity (mean difference $=0.241, p=0.044$ ), peer (mean difference $=0.226$, $p=0.032$ ) and prosocial (mean difference $=0.385, p<0.001$ ) subscales.

In the 15-16 years age group, girls reported a mean score of $3.2( \pm 1.832)$ for emotional symptoms compared to $2.62( \pm 1.822)$ among boys $(p=0.005)$. Other mean score differences which were significant in the total sample was not significant in this 15-16 year group. However, in the 12-14 years age group, conduct, peer and total difficulty scores were significantly higher among girls (Table 2). 
Normative banding and the cutoff values for the Sinhalese version of the SDQ were determined based on the distribution of raw data in this non-clinical sample [10]. Cutoff values between normal and borderline bands in the total difficulty score was set at 80th percentile and between borderline and abnormal bands at 90 th percentile
(Table 3). The objective was to detect the $10 \%$ of the population as abnormal scores and another $10 \%$ as borderline scores. However, due to the discrete nature of data obtained in the SDQ, the actual percentages categorised into these groups were slightly different from the expected percentages (Table 3).

Table 1. Scale means and gender of the Sinhalese version of the self reported SDQ

\begin{tabular}{lcccc}
\hline & Total $(n=1180)$ mean $(S D)$ & Boys $(n=535)$ mean(SD) & Girls $(n=645)$ mean(SD) & Significance \\
\hline Total score & $10.33(5.117)$ & $9.93(4.671)$ & $10.66(5.440)$ & $.014^{*}$ \\
Emotional symptoms & $2.85(1.971)$ & $2.70(1.915)$ & $2.97(2.009)$ & $.017^{*}$ \\
Conduct problems & $1.96(1.614)$ & $1.78(1.406)$ & $2.11(1.755)$ & $<.001^{*}$ \\
Hyperactivity & $3.37(1.822)$ & $3.41(1.808)$ & $3.34(1.835)$ & .503 \\
Peer problems & $2.15(1.602)$ & $2.04(1.383)$ & $2.24(1.760)$ & $.037^{*}$ \\
Prosocial symptoms & $7.98(1.666)$ & $8.03(1.503)$ & $7.93(1.789)$ & .362 \\
\hline
\end{tabular}

*mean difference between girls and boys was significant

Table 2. Scale means and gender effects by age group of Sinhalese version of self reported SDQ

\begin{tabular}{lcccccc}
\hline & \multicolumn{3}{c}{$\begin{array}{c}12-14 \text { year age group } \\
\text { Boys }(n=391) \\
\text { Mean }(S D)\end{array}$} & $\begin{array}{c}\text { Girls }(n=450) \\
\text { Mean }(S D)\end{array}$ & $p$ & \multicolumn{3}{c}{$\begin{array}{c}\text { 15-16 year age group } \\
\text { Boys }(n=143)\end{array}$} & $\begin{array}{c}\text { Girls }(n=178) \\
\text { Mean }(S D)\end{array}$ & $p$ \\
\hline Emotional & $2.72(1.948)$ & $2.92(2.072)$ & 0.135 & $2.62(1.822)$ & $3.20(1.832)$ & $0.005^{*}$ \\
Conduct & $1.71(1.424)$ & $2.19(1.822)$ & $0.000^{*}$ & $1.95(1.350)$ & $1.95(1.588)$ & 0.992 \\
Hyperactivity & $3.30(1.807)$ & $3.33(1.868)$ & 0.820 & $3.70(1.792)$ & $3.45(1.760)$ & 0.211 \\
Peer problems & $2.05(1.347)$ & $2.36(1.762)$ & $0.004^{*}$ & $2.02(1.475)$ & $1.97(1.750)$ & 0.766 \\
Prosocial & $7.97(1.477)$ & $7.78(1.791)$ & 0.103 & $8.17(1.570)$ & $8.32(1.690)$ & 0.429 \\
Total score & $9.78(4.692)$ & $10.81(5.610)$ & $0.004^{*}$ & $10.29(4.613)$ & $10.56(4.995)$ & 0.621 \\
\hline
\end{tabular}

*gender effect is significant

Table 3. Proposed normative bandings of Sinhalese version of self reported SDQ, compared with UK cut off values

\begin{tabular}{|c|c|c|c|c|c|c|}
\hline & \multicolumn{2}{|c|}{ Normal range } & \multicolumn{2}{|c|}{ Borderline range } & \multicolumn{2}{|c|}{ Abnormal range } \\
\hline & $S L(U K)$ & Exact \% & $S L(U K)$ & Exact \% & $S L(U K)$ & Exact \% \\
\hline Emotional & $0-4(0-5)$ & 82.8 & 5-6 (6) & 7.8 & $7-10(7-10)$ & 9.4 \\
\hline Conduct & $0-3(0-3)$ & 84.8 & $4(4)$ & 6.7 & $5-10(5-10)$ & 8.5 \\
\hline Hyperactivity & $0-5(0-5)$ & 80.8 & $6(6)$ & 13.8 & $7-10(7-10)$ & 5.4 \\
\hline Peer problems & $0-3(0-3)$ & 87.9 & $4(4-5)$ & 7.2 & $5-10(6-10)$ & 4.9 \\
\hline Prosocial & $7-10(6-10)$ & 80.2 & $6(5)$ & 10.7 & $0-5(0-4)$ & 9.1 \\
\hline Total score & $0-15(0-13)$ & 83.6 & $16-18(16-19)$ & 8.6 & $19-20(20-40)$ & 7.8 \\
\hline
\end{tabular}


Other than conduct and hyperactivity subscale, all other normative bands had different cut off points to the UK data [11]. These cut off points were on the higher side than the UK, reflecting the effect of higher mean scores. Separate analyses were conducted for boys and girls (not presented) which confirmed the requirement of higher cut off levels for girls.

PCA with varimax rotation was used to study the factor structure. Item list is rearranged for better understanding of factor loading after rotation. In the total sample, maximum variance explained by the factor 1 was $13.3 \%$ and the extracted factor consisted of all conduct scale items except “tempers”. Factor 2 was loaded with all items in the original emotional subscale and the loading values were more than .445 for all five items. However, three items from peer subscale and one from hyperactivity subscale were also loaded to this factor. None of the other three extracted factors contained more than 3 items from one subscale (Table 4).

Internal consistency (Cronbach’s alpha) for total difficulties was satisfactory in the total sample as well as among boys and girls separately (Table 5). However, in five subscales, homogeneity was low. In the total sample, Chrobach's alpha was lower than six in all subscales. Reliability of peer problems among boys was very poor. Girls reported higher reliability values ranging from 0.569 to 0.624 in all subscales.

Table 4. Extracted factor structure of the Sinhalese version of self reported SDQ 5-factor solution

\begin{tabular}{|c|c|c|c|c|c|}
\hline & Factor 1 & Factor 2 & Factor 3 & Factor 4 & Factor 5 \\
\hline Variance explained after rotation & $13.3 \%$ & $9.2 \%$ & $7.7 \%$ & $7.2 \%$ & $6.3 \%$ \\
\hline Item 3 : somatic symptoms & & .445 & & & \\
\hline Item 8 : worries & & .600 & & & \\
\hline Item 13 : unhappy & .360 & .488 & & & \\
\hline Item $16:$ nervous in new situations & & .572 & & & \\
\hline Item 24 : many fears & & .653 & & & \\
\hline Item 5 : tempers & & & .471 & & \\
\hline Item 7 : obedient & .311 & & & .485 & \\
\hline Item 12 : fights or bullies & .745 & & & & \\
\hline Item $18:$ lies or cheats & .425 & & .301 & & \\
\hline Item 22 : steals & .817 & & & & \\
\hline Item $2:$ restless & & & .712 & & \\
\hline Item 10 : fidgety & & & .705 & & \\
\hline Item 15 : easily distracted & & .321 & .409 & .377 & \\
\hline Item 21 : thinks before acting & & & & .556 & \\
\hline Item 25 : good attention & & & & .720 & \\
\hline Item 6 : solitary & & .417 & & & \\
\hline Item 11 : has good friend & .501 & & & & \\
\hline Item 14 : generally liked & & .325 & & & -.384 \\
\hline Item 19 : picked on or bullied & .543 & .335 & & & \\
\hline Item 23 : better with adults & & & & -.409 & -.342 \\
\hline Item 1 : considerate & & & & & .691 \\
\hline Item 4 : shares readily & -.603 & & & & \\
\hline Item 9 : helpful & & & & & .532 \\
\hline Item 17 : kind to younger & & & & & .475 \\
\hline Item 20 : often volunteers & -.645 & & & & \\
\hline
\end{tabular}

(only rotated loadings with absolute values $p>0.3$ are reported here)

Rotation method- Varimax with Kaiser Normalisation

Rotation converged in 12 iterations 


\section{Table 5. Internal consistency (Conbach's Alpha) of the Sinhalese version of the self reported SDQ}

\begin{tabular}{lc}
\hline SDQ scale & Conbach's Alpha \\
\hline Emotional & .594 \\
Conduct & .583 \\
Hyperactivity & .560 \\
Peer problems & .473 \\
Pro-social & .568 \\
Total score & .777 \\
\hline
\end{tabular}

All five subscales showed significant interaction with each other (Table 6). Highest interaction was reported in hyperactivity and conduct $(\mathrm{r}=0.474)$. Emotional subscale had Spearman's $r$ value more than 0.37 with conduct, hyperactivity and peer sub scales.

Table 6. Interaction of subscales for the Sinhalese version of the self reported SDQ

\begin{tabular}{lrrrr}
\hline & $\begin{array}{c}\text { Emotional } \\
\text { symptoms }\end{array}$ & $\begin{array}{c}\text { Conduct } \\
\text { problems }\end{array}$ & $\begin{array}{c}\text { Hyperactivity/ } \\
\text { inattention }\end{array}$ & $\begin{array}{c}\text { Peer } \\
\text { problems }\end{array}$ \\
\hline $\begin{array}{l}\text { Conduct } \\
\text { problems }\end{array}$ & $.370^{* *}$ & & & \\
$\begin{array}{l}\text { Hyperactivity/ } \\
\text { inattention }\end{array}$ & $.378^{* *}$ & $.474^{* *}$ & & \\
Peer problems & $.364^{* *}$ & $.296^{* *}$ & $.215^{* *}$ & \\
Prosocial & $-.156^{* *}$ & $-.364^{* *}$ & $-.289^{* *}$ & $-.296^{* *}$ \\
\hline
\end{tabular}

Spearman's correlation consistency coefficient

** Correlation is significant at the 0.01 level (2-tailed)

\section{Discussion}

Psychometric properties of the self reported Sinhalese version of SDQ evaluated using a national representative non-clinical sample is presented in this paper. The factor analysis and the internal consistency reported from the present study raises several problems related to the Sinhalese version of the SDQ that is available in the SDQ website.

The factor analysis of the Sinhalese version did not support the proposed five factor subscales of SDQ. In the total sample, all four items from emotional problem subscale and "I am usually on my own" from peer problem subscale exclusively loaded to a single factor. In addition, the remaining item from emotional subscale (unhappy) an item from hyperactive-inattention problem subscale and two other items from peer problem subscale (generally liked / picked on bullied) also loaded to this factor, but not exclusively. None of the other factors represented original subscales of SDQ with all five listed items. Four items in conduct problem subscale were loaded to a single factor together with, "has good friends" and "picked on or bullied" from peer problem scale, "shares readily" and "often volunteers" from prosocial problem subscale and "unhappy" from emotional subscale. This factor loading was not consistent with previously published data from other countries.

In 2001 Goodman reported the factor structure of self reported SDQ among 3983 children in which all items loaded primarily and mostly exclusively on predicted five subscales [12]. Most of the cross cultural studies confirm the reliability and validity of the five subscales in SDQ through component analysis. There were few exceptions to the generally accepted factor structure in some cultures. Factor structure of the Arabic version of SDQ showed that emotional, peer relationship and total scores are more heterogeneous or multifactorial. They concluded that further studies need to identify and use indigenously meaningful constructs. In a sample of 9,574 US children, the predicted five factor structure was not confirmed [13]. The authors showed that the US version fitted to a three factor model which consists of externalisation problems (conduct and hyperactive-inattention subscales), internalisation problems (emotional subscale) and positively construal factor. This proposed three factor structure was also observed in Finish version of SDQ [14]. A four factor solution was proposed in the Dutch version [15]. A six factor solution was proposed by another US study with five original subscales and positive construal additional factors. In our sample the factor structure was not compatible with any of these studies.

The internal consistency also raises problems about the reliability of the Sinhalese version of the SDQ. Total scale shows a satisfactory level of reliability (Chronbach's alpha 0.777) whereas all the subscales failed to achieve the level of reliability and the values were less than 0.6.

Goodman explained that in the SDQ, internalisation problems and externalisation problems are "uncontaminated" and it was proven in large community based samples in USA and China $[13,16]$. However, there is very little cross scale correlation. In our sample the cross scale correlation between hyperactive-inattention and conduct subscales (0.474) was higher than interaction of those two with emotional subscale. Interactions between internalization and externalization subscales also reached 0.37 , which is higher than previous studies. This is expected in our study as the emotional subscale item "unhappy" was loaded with conduct items and the hyperactive-inattention item "easily distracted", loaded to the emotional factor.

These observed, unsatisfactory psychometric properties of the Sinhalese version of SDQ could be due to socio-cultural differences in interpreting the items in SDQ. These questionnaires need proper cultural adaptations, rather than translation and back translation [17]. 
Adolescent behaviours, values, perceived socially accepted behaviours and judgment of own behaviours, heavily depend upon the culture. Interpretation of same statements could vary widely across cultures due to these effects. The use of the current version of the Sinhalese SDQ should be done with the understanding of the limitations that we observed in the reliability and factor structure. In addition, the version in SDQ website has inaccurate statements that need to be corrected.

For future references we reported the normative data and appropriate cut off points based on the raw values, which illustrated the need for different cut off values for the Sri Lankan population. Nevertheless, we did not discuss these values as there are issues in using this version of SDQ. Instead, we recommend further studies of the Sinhalese version of SDQ and re-wording of items, cultural adaptation of the questionnaire and revalidation to capture the intended construct of the original item.

\section{References}

1. Goodman R. The Strengths and Difficulties Questionnaire: a research note. The Journal of Child Psychology and Psychiatry 1997; 38: 581-6.

2. Downloadable SDQs and related items. 2009; Available from: http://www.sdqinfo.com/py/doc/b0.py. (Accessed October 2011)

3. Prior M, Virasinghe S, Smart D, Behavioural problems in Sri Lankan schoolchildren: associations with socio-economic status, age, gender, academic progress, ethnicity and religion. Social Psychiatry and Psychiatric Epidemiology 2005; 40: 654-62.

4. Perera H. Mental health of adolescent school children in Sri Lanka - a national survey. Sri Lanka Journal of Child Health 2004; 33: 78-81.

5. Agampodi TC, Agampodi SB, Fonseka P. Prevalence of mental health problems in adolescent school children in Galle District, Sri Lanka: Eight months after tsunami. Asia Pacific Journal of Public Health 2011; 23: 588-600.

6. Jayasinghe A. Child mental health problems in Hambantota District, Sri Lanka 2010, World Health Organization: Colombo. 1-101.
7. Department of Census and Statistics. Estimated mid year population by age and sex - 2000-2011 Available from: http:/ /www.statistics.gov.lk/page.asp?page=Population \%20and\%20Housing. (Accessed 22 October 2011).

8. UNICEF, Education statistics: Sri Lanka. Monitoring the situation of children and women. UNICEF 2008.

9. Ministry of Education, Sri Lanka. Number of schools by medium 2010-2011. Available from: http://www.moe.gov.lk/ web/index.php. (Accessed October 2011).

10. Woerner W, Becker A, Rothenberger A. Normative data and scale properties of the German parent SDQ. European Child and Adolescent Psychiatry 2004; 13: ii3-ii10.

11. SDQ: Normative SDQ Data from Britain. 2009; Available from: http://www.sdqinfo.com/UKNorm.html. (Accessed October 2011).

12. Goodman R. Psychometric properties of the strengths and difficulties questionnaire (SDQ). Journal of the American Academy of Child \& Adolescent Psychiatry 2001; 40: 1337-45.

13. Dickey WC, Blumberg SJ. Revisiting the factor structure of the strengths and difficulties questionnaire: United States, 2001. Journal of the American Academy of Child and Adolescent Psychiatry 2004; 43: 1159-67.

14. Koskelainen M, Sourander A, Vauras M. Self-reported strengths and difficulties in a community sample of Finnish adolescents. European Child and Adolescent Psychiatry 2001; 10: $180-5$.

15. Muris P, Meestars C, Eijkelenboom A, Vincken M. The self-report version of the Strengths and Difficulties Questionnaire: its psychometric properties in 8- to 13-yearold non-clinical children. British Journal of Clinical Psychology 2004; 43: 437-48.

16. Yao, S, Zhang C, Zhu X, Jing X, McWhinmie CM, Abela JRZ. Measuring adolescent psychopathology: psychometric properties of the self-report strengths and difficulties questionnaire in a sample of Chinese adolescents. Journal of Adolescent Health 2009; 45: 55-62.

17. Sumathipala A, Murray J. New approach to translating instruments for cross-cultural research: a combined qualitative and quantitative approach for translation and consensus generation. International Journal of Methods in Psychiatric Research 2000; 9: 87-95. 